\title{
Minor allele of rs12976445 polymorphism in the promoter region of microRNA-125a is associated with the severity of primary open-angle glaucoma (POAG)
}

Type

Research paper

\section{Keywords}

inflammation, POAG, miR-125a, IL-6R, rs12976445

\begin{abstract}
Introduction

The signaling pathway of IL- 6 has been reported to be associated with the severity of glaucoma. And the rs 12976445 SNP in miR-125 could alter the expression of miR-125, which is directly targeted by IL-6R.

Material and methods

In this study, we recruited 88 POAG patients and grouped them according to their genotype of rs 12976445 as GG group, GC group and CC group to study the association between the miR-125a polymorphism and POAG. We collected demographic characteristics and peripheral blood samples from 88 subjects. Then, rs 12976445 genotypes in these subjects were determined to evaluate their relationship with the POAG index. THP-1 and U937 cells were transfected with miR-125a mimic or IL-6R siRNA to assess the relationship between miR-125a and IL-6R/ACHE.

\section{Results}

IL-6R is a downstream target of miR-125a and the overexpression of miR-125a showed significantly decreased mRNA and protein levels of IL-6R. The expression levels of miR-125a were the highest in the GG group and the lowest in the CC group, while the activity of IL- 6 was comparable in the three groups. Moreover, the mRNA and protein levels of IL-6R were the lowest in the GG group and the highest in the CC group. Additionally, significantly thinner RNFL, larger average cup disc ratio, larger vertical cup disc ratio, and depressed visual field were observed in POAG patients carrying the CC genotype.
\end{abstract}

\section{Conclusions}

In summary, our data suggested that the rs 12976445 polymorphism was significantly associated with the risk of POAG. 
1 Minor allele of rs12976445 polymorphism in the promoter region of microRNA-125a is associated with the severity of primary open-angle glaucoma (POAG)

3 Wenjia Zhang ${ }^{1}$, Yan Li ${ }^{1}$, Hongqin Ke ${ }^{1}$, Yingting Wang ${ }^{1}$, Cong Duan ${ }^{1}$, Qin Zhu ${ }^{1}$, Hai Liu ${ }^{{ }^{*}}$

4 1. Department of Ophthalmology, The Second People's Hospital of Yunnan Province, $5 \quad$ Kunming, Yunnan Province, China 650031

$6 \quad$ *Corresponding author: Hai Liu

7 Institution: Department of Ophthalmology, The Second People's Hospital of Yunnan

8 Province, Kunming, Yunnan Province, China 650031

9 Address: No.176 Qingnian Rd, Kunming, Yunnan Province, China 650031

10 Email: docophthalo@yeah.net

\section{Abstract}

12 The signaling pathway of IL-6 has been reported to be associated with the severity of 13 glaucoma. And the rs12976445 SNP in miR-125 could alter the expression of miR-125, 14 which is directly targeted by IL-6R. In this study, we recruited 88 POAG patients and 15 grouped them according to their genotype of rs12976445 as GG group, GC group and CC 16 group to study the association between the miR-125a polymorphism and POAG. We 17 collected demographic characteristics and peripheral blood samples from 88 subjects. 18 Then, rs12976445 genotypes in these subjects were determined to evaluate their 19 relationship with the POAG index. THP-1 and U937 cells were transfected with miR-125a 20 mimic or IL-6R siRNA to assess the relationship between miR-125a and IL-6R/ACHE. IL-6R 21 is a downstream target of miR-125a and the overexpression of miR-125a showed 22 significantly decreased mRNA and protein levels of IL-6R. The expression levels of miR23 125a were the highest in the GG group and the lowest in the CC group, while the activity 24 of IL-6 was comparable in the three groups. Moreover, the mRNA and protein levels of IL$256 \mathrm{R}$ were the lowest in the GG group and the highest in the CC group. Additionally, 26 significantly thinner RNFL, larger average cup disc ratio, larger vertical cup disc ratio, and 
27 depressed visual field were observed in POAG patients carrying the CC genotype. In

28 summary, our data suggested that the rs12976445 polymorphism was significantly

29 associated with the risk of POAG.

30 Running title: rs12976445 is associated with severity of POAG

31 Keywords: POAG, miR-125a, IL-6R, inflammation, rs12976445

\section{Abbreviation}

33 POAG: primary open-angle glaucoma;

34 PBMC: peripheral blood monocyte;

35 ACHE: acetylcholinesterase;

36 RNFL: retinal nerve fiber layer;

37 IL-6R: interleukin-6 receptor;

38 RA: rim area;

39 C/D: cup/disc.

\section{Introduction}

41 As a kind of optic neuropathy featured by the loss of ganglion cells in the retina, glaucoma

42 is a leading contributor of blindness. As the most frequently diagnosed type of glaucoma,

43 primary open-angle glaucoma (POAG) impacts the lives of more than 40 million patients

44 worldwide ${ }^{1}$. More importantly, the visual impairment induced by POAG is irreversible,

45 making the early diagnosis of POAG an urgent need in its treatment ${ }^{2}$. It was previously

46 shown that the inflammation of the trabecular meshwork accelerates the progression of

47 POAG, suggesting that the oxidative stress and inflammation of the conjunctival stroma

48 play an essential role in the diagnosis and treatment of POAG ${ }^{2,3}$.

49 MicroRNAs (miRNAs) have been detected in many biological species and can regulate the

50 expression of their target mRNAs at the post-transcriptional level by interacting with the

513 ' untranslated region (3' UTR) of these mRNAs, thus participating in the pathogeneses of 
52 many diseases ${ }^{4}$. As a miRNA highly expressed in many types of mammalian cells, miR-125 53 has three homologs, i.e., miRNA-125b-2, miRNA-125b-1 and miR-125a. In particular, 54 miRNA-125b-2 was recently demonstrated to be implicated in various immune reactions $55 \quad 5,6$. MiRNA-125b-2 has also been shown to play important roles in stabilizing the activities 56 of signal transducer and activator of transcription 3 (STAT3) in antigen-presenting cells ${ }^{7}$. 57 The overexpression of miR-125a reduced the expression of various pro-inflammatory 58 cytokines such as IL-12, p40, IL-6, and TNF- $\alpha$ in human monocytes ${ }^{8}$. MiR-125a can also 59 inhibit the polarization of M1 macrophages ${ }^{9-11}$.

60 Past experiments demonstrated that the rs12976445 single nucleotide polymorphism 61 (SNP) in pre-miR-125a can affect the maturation of pre-miR-125a ${ }^{12}$. Past studies also 62 demonstrated that the rs12976445 SNP can affect receptor tyrosine-protein kinase erbB632 (ERBB2) expression in patients with breast cancer. As a miR-125a target, ERBB2 64 expression is increased in patients with esophageal cancer 13, 14. Existing data also 65 suggested that the rs12976445 SNP T allele affects the maturation of miRNA-125a, 66 leading to increased susceptibility to autoimmune disorders ${ }^{12,15}$. Furthermore, the 67 genotypes of rs12976445 SNP also regulate miRNA-125a expression and the expression 68 of its target against decapentaplegic homolog 2 (SMAD2) and transforming growth factor69 beta 1 (TGFB1).

70 Both interleukin-6 receptor (IL-6R) and interleukin-6 (IL-6) have been linked to POAG71 induced autoimmune disorder ${ }^{16}$. In addition, upon the increased intraocular pressure in 72 POAG patients, IL-6R expression in the trabecular meshwork also increases, suggesting 73 that the allele frequency, as well as the genotypes of IL-6R and IL-6 are apparently 74 affected by the onset of POAG ${ }^{16,17}$. Moreover, the serum levels of IL-6 in POAG are 75 decreased along with an apparently elevated level of fibrinogen, which induces a high 76 level of hemorheological viscosity ${ }^{16}$.

77 The deregulation of the IL-6 signaling pathway is associated with the severity of glaucoma, 78 while IL-6R is a direct target of miR-125a ${ }^{18}$. Furthermore, rs12976445 SNP located in miR79125 has been shown to alter the expression of miR-125 ${ }^{19}$. In this study, we hypothesized 
that the rs12976445 polymorphism could be associated with the severity of POAG, which

81 enables miR-125a to be a potential biomarker for the susceptibility to POAG. Accordingly,

82 we collected blood and peripheral blood monocyte (PBMC) samples from POAG subjects

83 and studied the association between the polymorphism in miR-125a and the severity of

84 POAG.

\section{Materials and Methods}

\section{Clinical data of patients and sample collection}

87 This study enrolled a total of 88 POAG patients. After enrollment, peripheral blood 88 samples were collected from each POAG subject and the genotype of rs12976445 in each 89 sample was analyzed by Taqman genotyping assays to determine its genotype of 90 rs12976445. At the same time, the clinical-pathological data, demographic characteristics 91 and PBMC samples were collected from all POAG subjects. Among these 88 POAG patients, 9235 POAG subjects (the GG group, N=35) carried the GG genotype of rs12976445, 28 POAG 93 subjects (the GC group, $\mathrm{N}=28$ ) carried the GC genotype of $\mathrm{rs} 12976445$, and 25 POAG 94 subjects (the CC group, $\mathrm{N}=25$ ) carried the CC genotype of rs12976445. No significant 95 difference in respect to age and gender were spotted after these randomly-selected 96 patients were grouped (Page $>0.05, \mathrm{P}$ gender $>0.05$ ). According to the studies by 97 Lehmann et al. ${ }^{20}$, rs12976445 might not a somatic tumor-origin mutation since its ratio 98 varies in different study populations. Generally, the ratio of some genotype group might 99 by rare in the general population. However, since our study was a functional study with a 100 relatively small sample size instead of an association study, to balance the sample size of 101 each genotype group, we recruited a comparable number of participants in each group. 102 The presence of systemic diseases (hypertension, diabetes mellitus and hyperlipidemia) 103 also did not differ among the subjects in the three groups ( $P>0.05)$. All patients have 104 signed written informed consent before the study begins.

105 Calculation of the cup-disc (C/D) ratio using a method based on slit-lamp 106 ophthalmoscopy 
107 In this study, the C/D ratio of each POAG subject was independently calculated by 3 108 experienced clinicians specialized in the treatment of POAG. The determination of the

$109 \mathrm{C} / \mathrm{D}$ ratio was done using a method based on slit-lamp ophthalmoscopy by scanning the 110 optic nerve head in each POAG patient. The severity of POAG in these subjects was 111 classified as mild or severe depending on their C/D ratio measurement. In brief, mild 112 POAG indicated the POAG patients were in the early to medium stage of the disease and 113 their $C / D$ ratio was $\geq 0.3$ and $\leq 0.7$. On the other hand, severe POAG indicated the POAG 114 patients were in the advanced stage of the disease and their $C / D$ ratio was $\geq 0.7$ and $\leq 1.0$. 115 At the same time, alterations of the optic nerve disc were also assessed. During the 116 determination of the severity of POAG and the assessment of the optic nerve disc, the 3 117 clinicians would cast their votes respectively and the final results were obtained via 118 consensus.

\section{Genotyping using Taqman assay}

120 The genotypes of rs12976445 SNP, i.e., genotypes GG, GC and CC, were determined using 121 a Taqman method. First, genomic DNA was separated from collected PBMC samples using 122 a DNA extraction kit (Tiangen, Beijing, China) following the standard protocol provided by 123 the manufacturer. Then, the isolated DNA samples were amplified on an ABI 7300 Real 124 Time PCR instrument (Applied Biosystems, Foster City, CA) using a Taqman method to 125 determine the genotype of rs12976445 SNP in each sample.

\section{ELISA assay}

127 The concentration of IL-6 in peripheral blood samples collected from each POAG subject 128 was assayed using an IL-6 ELISA assay kit purchased from Bio-Rad Laboratory (Hercules, 129 CA) and the assay was carried out per the standard protocol provided on kit instruction.

\section{RNA isolation and real-time PCR}

131 First, total RNA in collected peripheral blood samples and PBMC samples from each POAG 132 patient as well as in cultured THP-1 and U937 cells was extracted using a Trizol 133 experimental assay (Invitrogen, Carlsbad, CA) according to the standard method 
recommended by the manufacturer. In the next step, the isolated RNA was converted to cDNA using a Reverse Transcription Kit (Thermo Fisher Scientific, Waltham, MA) before the relative expression of miR-125a, IL-6R mRNA as well as acetylcholinesterase (ACHE) mRNA in each sample was detected using Taqman Universal Master Mix (Applied Biosystems, Foster City, CA) on the ABI 7300 Real Time PCR instrument (Applied Biosystems, Foster City, CA). The quantification of miR-125a, IL-6R mRNA as well as ACHE mRNA was carried out using the standard $2^{-\triangle \Delta C T}$ method, while the expression of GAPDH and U6 was used as the internal standard for miR-125a and IL-6R/ACHE mRNA, respectively. The sequence of the primer pairs used are: miR-125a-F: 5'CCTGAGACCCTTTAACC -3'; miR-125-R: 5'- GAACATGTCTGCGTATCTC -3'; IL-6R-F: 5'GACTGTGCACTTGCTGGTGGAT -3'; IL-6R-R: 5' - ACTTCCTCACCAAGAGCACAGC -3'; ACHE-F: 5'- GTTCTCCTTCGTGCCTGTGGTA -3'; ACHE-R: 5'- ATACGAGCCCTCATCCTTCACC -3'; 146 GAPDH-F: 5'- GTCTCCTCTGACTTCAACAGCG - 5'; $^{\prime}$ GAPDH-R: 5'147 ACCACCCTGTTGCTGTAGCCAA -3'; U6-F: 5'- CTCGCTTCGGCAGCACAT -3'; U6-R: 5'TTTGCGTGTCATCCTTGCG -3'.

\section{Cell culture and transfection}

150 THP-1 and U937 cells were acquired from the American Type Culture Collection (ATCC, 151 Manassas, VA) and stored in a liquid nitrogen tank. Prior to the experiment, the cells were 152 thawed and passaged in Dulbecco's Modified Eagle Medium (DMEM, Gibco, Thermo 153 Fisher Scientific, Waltham, MA) added with $10 \%(\mathrm{v} / \mathrm{v})$ fetal bovine serum (FBS, Gibco, 154 Thermo Fisher Scientific, Waltham, MA) and appropriate concentrations of penicillin and 155 streptomycin (Sigma Aldrich, St. Louis, MO). The cells were incubated in a regular tissue

156 culture incubator containing $5 \% \mathrm{CO}_{2}$ and $95 \%$ air. The culture conditions were saturated 157 humidity and $37^{\circ} \mathrm{C}$. The cells were passaged once every 2 days using trypsin-EDTA (Gibco, 158 Thermo Fisher Scientific, Waltham, MA) under they reached logarithmic growth. Then, 159 the cells were randomly divided into the following 3 groups: 1 . the scramble control group, 160 in which the cells were treated with PBS during the subsequent transfection experiment; 161 2. the group of miR-125a mimics, in which the cells were transfected with miR-125a 162 mimics using Lipofectamine 3000 transfection reagent (Invitrogen, Carlsbad, CA) 
163 following the standard procedure provided by the manufacturer; 3 . the group of IL-6R 164 siRNA, in which the cells were transfected with IL-6R siRNA using the Lipofectamine 3000 165 transfection reagent. At 48 after transfection, the cells were harvested to assay the 166 expression of target genes.

\section{Vector construction, mutagenesis and luciferase assay}

168 To determine the effect of miR-125a on the expression of IL-6R and ACHE, the 3' UTRs of 169 IL-6R and ACHE mRNAs carrying the miR-125a binding sites were respectively inserted 170 into pcDNA vectors (psiCHECK ${ }^{\mathrm{TM}}-1$, Promega, Madison, WI) to create the plasmids of wild 171 type 3' UTRs of IL-6R and ACHE mRNAs. Then, site-directed mutagenesis was carried out 172 using a Quick Change mutagenesis kit (Stratagene, San Diego, CA) following the kit 173 instruction to induce site-directed mutations in the miR-125a binding sites located on the 174 3' UTRs of IL-6R and ACHE mRNAs, respectively. The full length 3' UTR of IL-6R and ACHE 175 mRNA carrying the mutant miR-125a binding sites were also inserted into separate pcDNA 176 vectors to create the plasmids of mutant 3' UTRs of IL-6R and ACHE mRNAs, respectively. 177 In the next step, THP-1 and U937 cells were co-transfected with the vectors of wild 178 type/mutant 3' UTRs of IL-6R or ACHE mRNA in conjunction with miR-125a or scramble 179 control using the Lipofectamine 3000 transfection agent. At 48 after transfection, the 180 luciferase activity of transfected cells was determined using a Bright-Glo luciferase assay 181 kit (Promega, Madison, WI).

\section{Western blot}

183 The protein expression of IL-6R and ACHE in collected peripheral blood and PBMC samples 184 from each POAG patient as well as in cultured THP-1 and U937 cells was determined using 185 a standard Western blot assay.

\section{Statistical analysis}

187 Categorical variables were tested using Chi Square tests, while continuous variables were 188 tested using Student's t tests. The categorical results were shown as percentages while 189 the continuous variables were shown in mean \pm standard deviations. For comparisons 
between multiple groups, one-way ANOVA was used with Tukey's test as the post hoc test. All statistical analyses were carried out using SPSS 21.0 (SPSS, Chicago, IL). The level

192 of statistical significance was set to $P<0.05$.

\section{Results}

\section{Validation of downstream targets of miR-125a}

195 To find potential targets of miR-125a, we searched the TargetScan, Pictar-Vert, and 196 Microrna.Org databases, and found IL-6R and ACHE as potential targets of miR-125a. As 197 shown in Fig.1A and Fig.1C, both IL-6R and ACHE carried a miR-125a binding site, 198 indicating that IL-6R and ACHE might be direct targets of miR-125a. To verify that the miR199 125a binding sites in IL-6R and ACHE were responsible for miR-125a regulation, we 200 constructed vectors containing wild-type or mutant IL-6R and ACHE directly fused to the 201 firefly luciferase gene. Then, the wild-type or mutant vectors of IL-6R/ACHE were co202 transfected into THP-1/U937 cells with miR-125a or miR-125a NC. The results showed 203 that the relative luciferase activity of miR-125a sharply decreased in cells transfected with 204 wild type IL-6R (Fig.1B). However, the relative luciferase activity of miR-125a displayed no 205 differences in cells either transfected with wild type ACHE or mutant type ACHE (Fig.1D). 206 Taken together, these findings indicated that IL-6R, but not ACHE, is a direct target for 207 miR-125a.

208 To further verify above results, THP-1/U937 cells were transfected with miR-125a mimic 209 or IL-6R siRNA. Then, RT-qPCR and Western blot were performed to evaluate the mRNA 210 and protein levels of IL-6R and ACHE in THP-1/U937 cells. As shown in Fig.2A and 3A, the 211 mRNA and protein levels of IL-6R in cells transfected with miR-125a mimic or IL-6R siRNA 212 were significantly decreased. Meanwhile, the mRNA and protein levels of ACHE (Fig.2B 213 and Fig.3B) showed no significant difference among the three groups in THP-1/U937 cells.

\section{Distribution of different genotypes of rs12976445 in POAG patients}

215 The peripheral blood samples of POAG patients were collected to determine their 216 genotypes of rs12976445. The demographic characteristics of subjects were described in 
217 Table 1. Among all POAG subjects, 35 cases carried the GG genotype of rs12976445, 28 218 cases carried the GC genotype of rs12976445, and 25 cases carried the CC genotype of 219 rs12976445. These groups were well matched for age and gender (Page $>0.05, P$ gender $>$ 220 0.05). The presence of systemic diseases (hypertension, diabetes mellitus and 221 hyperlipidemia) did not differ among the subjects in the three groups $(P>0.05)$.

222 Expression levels of miR-125a and IL-6R in the PMBC of POAG patients carrying different 223 genotypes of rs12976445

224 The peripheral blood samples collected from GG, GC and CC groups were analyzed using 225 RT-qPCR and ELISA. The expression level of miR-125a (Fig.4A) was the highest in the GG 226 group and the lowest in the CC group, while the serum activity of IL- 6 was comparable in 227 three groups (Fig.4B).

228 Subsequently, the mRNA level of miR-125a (Fig.5A) and the activity of IL-6R (Fig.5B) in 229 peripheral monocytes collected from the three groups were tested. As shown in Fig.5, the 230 results were consistent with those obtained using peripheral blood samples.

231 Moreover, the mRNA and protein levels of IL-6R and ACHE in peripheral blood samples of 232 POAG patients were detected by RT-qPCR and Western blot. Accordingly, the mRNA and 233 protein levels of IL-6R were the lowest in the GG group and the highest in the CC group 234 (Fig.6), while the mRNA and protein levels of ACHE were comparable among the three 235 groups (Fig.7).

236 Comparison of demographic and clinical data among POAG patients carrying the GG, GC and CC genotypes of rs12976445 SNP

238 The glaucoma indexes of POAG patients in the three groups were collected. As shown in 239 Fig.8, these groups were well matched on central corneal thickness (Fig.8A) and IOP 240 (Fig.8B) during enrollment. However, the POAG patients carrying the CC genotype of 241 rs12976445 SNP showed significant thinner retinal nerve fiber layer (RNFL) (Fig.8C), larger 242 vertical cup disc ratio (Fig.8E), larger average cup disc ratio (Fig.8F), and depressed visual 243 field. 
245 It has been indicated that the minor allele of rs12976445 apparently changes the ratio between mature miR-125a expression and pre-miR-125a expression, which indicated that rs12976445 could affect the maturation of miR-125a ${ }^{21}$. Moreover, miR-125a expression

248 in the TT group was similar to that in the CT group and was apparently elevated in the CC 249 group, suggesting a dominant role of rs12976445 minor allele ${ }^{19}$. In this study, the 250 glaucoma indexes of the POAG patients in the three groups indicated that the POAG 251 patients carrying the CC genotype of rs12976445 SNP showed significant thinner RNFL, 252 larger average cup disc ratio, larger vertical cup disc ratio, and depressed visual field. 253 Meanwhile, the expression level of miR-125a was the highest in the GG group and the 254 lowest in the CC group, which also led to the most suppressed mRNA and protein levels 255 of IL-6R in the GG group. Meanwhile, the mRNA and protein levels of ACHE were comparable among the three groups. Therefore, the above results all supported our 257 hypothesis that the allele type of rs12976445 apparently influenced the severity of POAG 258 via regulating expression of miR-125a and IR-6R.

259 Both miRNA-125b and miR-125a are members of the miRNA-125 family, which was shown 260 to play important roles in various processes such as the apoptosis, growth and 261 differentiation of cells ${ }^{22}$. MiR-125a is inhibited during inflammation, while miRNA-125b 262 can reduce inflammatory reactions by targeting TNF- $\alpha^{23-26}$. Nevertheless, a past study 263 demonstrated that miR-125a promotes the pro-inflammatory adaptation of macrophages 264 while increasing their response to IFN- $\alpha$ stimulation ${ }^{27}$. It was also shown that miR-125a 265 inhibits LPS-induced expression of TNF- $\alpha$, iNOS as well as IL-12, suggesting that while miR266 125a-5p can target TNF- $\alpha$, its anti-inflammatory role is mediated via other regulators. It 267 was also shown that KLF13, a transcriptional factor and a target of miR-125a-5p, can 268 inhibit inflammation and decrease the activation level of T cells ${ }^{28}$. In a past study, Graff 269 et al. showed that the over expression of miR-125a-5p induces the activation of THP-1 270 cells, while another report demonstrated that miR-125a-5p can activate NF-kB signaling 271 in cells of diffuse large B-cell lymphoma ${ }^{29,30}$. It was also demonstrated that miR-125a-5p 272 can mediate the IL-6-induced Treg cell sensitivity. In the absence of stimulation by IL-6, 
273 the change in miR-125a-5p expression failed to affect FOXP3 expression or Treg activity

274 31. The IL-6 signaling is crucial for iTreg differentiation. Nevertheless, altered miR-125a-5p

275 expression in naïve T cells exerted no effects on iTreg and naïve T cell polarization ${ }^{31}$. In

276 this study, IL-6R and ACHE were shown to contain miR-125a binding sites. The relative

277 luciferase activity of miR-125a sharply decreased in cells transfected with wild type IL-6R,

278 while the relative luciferase activity of miR-125a displayed no differences in cells either

279 transfected with wild type ACHE or mutant type ACHE. In addition, mRNA and protein

280 levels of IL-6R and ACHE were evaluated in THP-1 and U937 cells transfected with miR-

281 125a mimic or IL-6R siRNA. The mRNA and protein levels of IL-6R in cells transfected with

282 miR-125a mimic or IL-6R siRNA were significantly decreased, while the mRNA and protein

283 levels of ACHE showed no significant differences among the three groups.

284 Released from adipocytes, macrophages, as well as other types of cells such as fibroblasts, 285 skeletal muscle cells as well as endothelial cells, IL-6 plays important roles in the 286 regulation of lipid metabolism as well as body weight ${ }^{32-35}$. IL-6 is also involved in the 287 formation of obesity as well as insulin resistance. Nevertheless, the functions of IL-6 can 288 be complicated ${ }^{36-38}$. For example, IL-6 can play an anti-inflammatory role to block the 289 functions of TNF- $\alpha$, to promote the polarization of $M 2$ macrophages, as well as to alleviate 290 insulin resistance ${ }^{39,40}$. Other studies showed that obesity can elevate the level of IL-6 as 291 well as IL-6R in adipose tissues to elevate the levels of IP-10, MCP-1, as well as TNF- $\alpha$ in 292 these tissues ${ }^{41}$. Past studies also demonstrated that metabolic synthesis is a POAG risk 293 factor and is involved in alternating the allele frequency of certain genes. For example, 294 during the onset as well as development of POAG, the functions of various factors were 295 modified by metabolic synthesis, such as the effect of Serpine1 on the trabecular 296 meshwork, the effect of ENPP1 on the proliferation of cells in the trabecular meshwork, 297 the effects of IL-6R, IL-6, and E-Sel on autoimmune reactions, the effect of LIPC and FGB 298 on hyper-viscosity, as well as the effect of ADIPOQ on NOS/NO synthesis. Past studies also 299 showed that the expression of IL-6 in the serum of POAG patients is elevated ${ }^{42}$. In 300 addition, the $\mathrm{G}$ allele of the single nucleotide polymorphism (SNP) located at position (- 
301 174) of the IL-6 gene was shown to elevate the expression of IL-6 proteins in POAG 302 patients ${ }^{43}$.

303 However, despite the results obtained which supported our hypothesis, the conclusion 304 could be quite limited due to the small sample size recruited in this study. In this study, 305 only 88 POAG patients were recruited and subjected to genotyping, therefore, this limited 306 sample size will influence the accuracy of the correlation analysis between allele type and 307 POAG severity. In our future study, large sample size is necessary, preferably with varied 308 nations.

309 Conclusion

310 In summary, our data suggested that the rs12976445 polymorphism was significantly 311 associated with the risk of POAG. To our knowledge, this is the first study investigating 312 the association between miR-125a rs12976445 polymorphisms and POAG. The miR-125a 313 rs12976445 SNP may be used as a biomarker to determine the susceptibility to POAG 314 after further validation with larger scale population.

\section{Availability of data and material}

316 The data that support the findings of this study are available from the corresponding 317 author upon reasonable request.

\section{Competing interests}

319 The authors declare that they have no competing interests.

\section{$320 \quad$ Funding}

321 None

\section{Authors' contributions}

323 Wenjia Zhang and Hai Liu planned the study, Yan Li and Hongqin Ke collected the 324 literatures, Wenjia Zhang, Yan Li, Hongqin Ke, Yingting Wang and Cong Duan collected 
325 and analyzed the data, Qin Zhu and Hai Liu visualized the data, Wenjia Zhang and Hai Liu

326 composed the manuscript, and all the other co-authors approved the final manuscript.

\section{Acknowledgements}

328 Not applicable

\section{$329 \quad$ Figure legends}

330 Table1. Clinical pathological data of POAG patients carrying different genotypes of 331 rs12976445.

\section{$332 \quad$ Fig.1}

333 Luciferase assay confirmed the target genes of miR-125a in THP-1 and U937 cells (* P 334 value $<0.05$ vs. miR-NC group; number of replicas $=3$ )

335 A: predicted binding site of miR-125a in IL-6R

336 B: luciferase activities of miR-125a in THP-1 cells co-transfected with wild/mutant type of 337 IL-6R and miR-125a /NC.

338 C: predicted binding site of miR-125a in ACHE

339 D: luciferase activities of miR-125a in THP-1 cells co-transfected with wild/mutant type of 340 ACHE and miR-125a /NC.

\section{$341 \quad$ Fig.2}

342 mRNA and protein levels of IL-6R and ACHE in THP-1 cells transfected with NC, miR-125a 343 mimics or IL-6R siRNA (* P value < 0.05 vs. scramble control group; number of replicas = 344 3)

345 A: mRNA and protein levels of IL-6R in the three THP-1 groups.

346 B: mRNA and protein levels of ACHE in the three THP-1 groups.

$347 \quad$ Fig.3 
348 mRNA and protein levels of IL-6R and ACHE in U937 cells transfected with NC, miR-125a 349 mimics or IL-6R siRNA ( $*$ P value $<0.05$ vs. scramble control group; number of replicas = $3503)$

351 A: mRNA and protein levels of IL-6R in the three U937 groups.

352 B: mRNA and protein levels of ACHE in the three U937 groups.

\section{$353 \quad$ Fig.4}

354 Relative expression of miR-125a mRNA and activity of IL-6R in blood samples collected 355 from POAG patients carrying GG, GC, and CC genotypes of rs12976445 (* P value $<0.05$ 356 vs. GG group; ** P value < 0.05 vs. GC group; number of replicas $=3$ ).

357 A: Relative expression of miR-125a in the three groups.

358 B: IL-6 activity in the three groups.

$359 \quad$ Fig.5

360 Relative expression of miR-125a mRNA and activity of IL-6R in PBMC samples collected 361 from POAG patients carrying GG, GC, and CC genotypes of rs12976445 (* P value $<0.05$ 362 vs. GG group; $* *$ P value $<0.05$ vs. GC group; number of replicas $=3$ ).

363 A: Relative expression of miR-125a in the three groups.

364 B: IL-6 activity in the three groups.

365 Fig.6

366 mRNA and protein levels of IL-6R in PBMC samples collected from POAG patients carrying 367 GG, GC, and CC genotypes of rs12976445 (* P value < 0.05 vs. GG group; ** P value $<0.05$ 368 vs. GC group; number of replicas = 3).

369 A: mRNA levels of IL-6R in the three groups

370 B: protein levels of IL-6R in the three groups

$371 \quad$ Fig.7 
372 mRNA and protein levels of ACHE in PBMC samples collected from POAG patients carrying

373 GG, GC, and CC genotypes of rs12976445 (number of replicas = 3).

374 A: mRNA levels of ACHE in the three groups

375 B: protein levels of ACHE in the three groups

$376 \quad$ Fig.8

377 Comparison of demographic and clinical data among POAG patients carrying GG, GC and 378 CC genotypes of rs12976445 (* P value < 0.05 vs. GG group; ** P value < 0.05 vs. GC group;

379 number of replicas $=3$ ).

380 A: central corneal thickness in the three groups

381 B: IOP at recruitment in the three groups

382 C: retinal nerve fiber layer thickness in the three groups

383 D: rim area in the three groups

384 E: vertical cup disc ratio in the three groups

$385 \mathrm{~F}$ : average cup disc ratio in the three groups

386 G: mean deviation in the three groups

$387 \mathrm{H}$ : pattern SD in the three groups

\section{$388 \quad$ References}

389 1. Tham, Y. C.; Li, X.; Wong, T. Y.; Quigley, H. A.; Aung, T.; Cheng, C. Y., Global prevalence 390 of glaucoma and projections of glaucoma burden through 2040: a systematic review and meta391 analysis. Ophthalmology 2014, 121 (11), 2081-90.

392 2. Helin-Toiviainen, M.; Ronkko, S.; Kaarniranta, K.; Puustjarvi, T.; Rekonen, P.; Ollikainen, 393 M.; Uusitalo, H., Oxidized low-density lipoprotein, lipid and calcium aggregates reveal oxidative 394 stress and inflammation in the conjunctiva of glaucoma patients. Acta Ophthalmol 2017, 95 (4), $395378-385$.

$396 \quad$ 3. Izzotti, A.; La Maestra, S.; Micale, R. T.; Longobardi, M. G.; Sacca, S. C., Genomic and 397 post-genomic effects of anti-glaucoma drugs preservatives in trabecular meshwork. Mutat Res 398 2015, 772, 1-9.

399 4. Kalla, R.; Ventham, N. T.; Kennedy, N. A.; Quintana, J. F.; Nimmo, E. R.; Buck, A. H.; 400 Satsangi, J., MicroRNAs: new players in IBD. Gut 2015, 64 (3), 504-17. 
5. Shaham, L.; Binder, V.; Gefen, N.; Borkhardt, A.; Izraeli, S., MiR-125 in normal and malignant hematopoiesis. Leukemia 2012, 26 (9), 2011-8.

6. Long, H.; Yin, H.; Wang, L.; Gershwin, M. E.; Lu, Q., The critical role of epigenetics in systemic lupus erythematosus and autoimmunity. J Autoimmun 2016, 74, 118-138.

7. Hildebrand, D.; Eberle, M. E.; Wolfle, S. M.; Egler, F.; Sahin, D.; Sahr, A.; Bode, K. A.; Heeg, K., Hsa-miR-99b/let-7e/miR-125a Cluster Regulates Pathogen Recognition ReceptorStimulated Suppressive Antigen-Presenting Cells. Front Immunol 2018, 9, 1224.

8. Curtale, G.; Renzi, T. A.; Mirolo, M.; Drufuca, L.; Albanese, M.; De Luca, M.; Rossato, M.; Bazzoni, F.; Locati, M., Multi-Step Regulation of the TLR4 Pathway by the miR-125a 99b let7e Cluster. Front Immunol 2018, 9, 2037.

9. $\quad$ Banerjee, S.; Cui, H.; Xie, N.; Tan, Z.; Yang, S.; Icyuz, M.; Thannickal, V. J.; Abraham, E.; Liu, G., miR-125a-5p regulates differential activation of macrophages and inflammation. $J$ Biol Chem 2013, 288 (49), 35428-36.

10. Song, A.; Patel, A.; Thamatrakoln, K.; Liu, C.; Feng, D.; Clayberger, C.; Krensky, A. M., Functional domains and DNA-binding sequences of RFLAT-1/KLF13, a Kruppel-like transcription factor of activated T lymphocytes. J Biol Chem 2002, 277 (33), 30055-65.

11. Zhao, X.; Tang, Y.; Qu, B.; Cui, H.; Wang, S.; Wang, L.; Luo, X.; Huang, X.; Li, J.; Chen, S.; Shen, N., MicroRNA-125a contributes to elevated inflammatory chemokine RANTES levels via targeting KLF13 in systemic lupus erythematosus. Arthritis Rheum 2010, 62 (11), 3425-35.

12. Hu, Y.; Liu, C. M.; Qi, L.; He, T. Z.; Shi-Guo, L.; Hao, C. J.; Cui, Y.; Zhang, N.; Xia, H. F.; $\mathrm{Ma}, \mathrm{X}$. , Two common SNPs in pri-miR-125a alter the mature miRNA expression and associate with recurrent pregnancy loss in a Han-Chinese population. RNA Biol 2011, 8 (5), 861-72.

13. Kato, H.; Arao, T.; Matsumoto, K.; Fujita, Y.; Kimura, H.; Hayashi, H.; Nishiki, K.; Iwama, M.; Shiraishi, O.; Yasuda, A.; Shinkai, M.; Imano, M.; Imamoto, H.; Yasuda, T.; Okuno, K.; Shiozaki, H.; Nishio, K., Gene amplification of EGFR, HER2, FGFR2 and MET in esophageal squamous cell carcinoma. Int J Oncol 2013, 42 (4), 1151-8.

14. Fassan, M.; Pizzi, M.; Realdon, S.; Balistreri, M.; Guzzardo, V.; Zagonel, V.; Castoro, C.; Mastracci, L.; Farinati, F.; Nitti, D.; Zaninotto, G.; Rugge, M., The HER2-miR125a5p/miR125b loop in gastric and esophageal carcinogenesis. Hum Pathol 2013, 44 (9), 1804-10.

15. Inoue, Y.; Watanabe, M.; Inoue, N.; Kagawa, T.; Shibutani, S.; Otsu, H.; Saeki, M.; Takuse, Y.; Hidaka, Y.; Iwatani, Y., Associations of single nucleotide polymorphisms in precursormicroRNA (miR)-125a and the expression of mature miR-125a with the development and prognosis of autoimmune thyroid diseases. Clin Exp Immunol 2014, 178 (2), 229-35.

16. Zhou, G.; Liu, B., Single nucleotide polymorphisms of metabolic syndrome-related genes in primary open angle glaucoma. Int J Ophthalmol 2010, 3 (1), 36-42.

17. Diskin, S.; Kumar, J.; Cao, Z.; Schuman, J. S.; Gilmartin, T.; Head, S. R.; Panjwani, N., Detection of differentially expressed glycogenes in trabecular meshwork of eyes with primary open-angle glaucoma. Invest Ophthalmol Vis Sci 2006, 47 (4), 1491-9.

18. Bukhari, S. M.; Kiu, K. Y.; Thambiraja, R.; Sulong, S.; Rasool, A. H.; Liza-Sharmini, A. T., Microvascular endothelial function and severity of primary open angle glaucoma. Eye (Lond) 2016, 30 (12), 1579-1587.

19. Ma, J. F.; Zang, L. N.; Xi, Y. M.; Yang, W. J.; Zou, D., MiR-125a Rs12976445 Polymorphism is Associated with the Apoptosis Status of Nucleus Pulposus Cells and the Risk of Intervertebral Disc Degeneration. Cell Physiol Biochem 2016, 38 (1), 295-305.

20. Lehmann, T. P.; Korski, K.; Ibbs, M.; Zawierucha, P.; Grodecka-Gazdecka, S., \& Jagodziński, P. P. (2013). rs12976445 variant in the pri-miR-125a correlates with a lower level of hsa-miR-125a and ERBB2 overexpression in breast cancer patients. Oncology letters, 5(2), 569-573. https://doi.org/10.3892/ol.2012.1040. 
21. Gao, X.; Yang, L.; Ma, Y.; Yang, J.; Zhang, G.; Huang, G.; Huang, Q.; Chen, L.; Fu, F.;

450 Chen, Y.; Su, D.; Dong, Y.; Ma, X.; Lu, C.; Peng, X., No association of functional variant in pri-miR451218 and risk of congenital heart disease in a Chinese population. Gene 2013, 523 (2), 173-7.

452 22. Sun, Y. M.; Lin, K. Y.; Chen, Y. Q., Diverse functions of miR-125 family in different cell 453 contexts. J Hematol Oncol 2013, 6, 6.

454 23. Murphy, A. J.; Guyre, P. M.; Pioli, P. A., Estradiol suppresses NF-kappa B activation 455 through coordinated regulation of let-7a and miR-125b in primary human macrophages. $J$ Immunol 2010, 184 (9), 5029-37.

24. Rajaram, M. V.; Ni, B.; Morris, J. D.; Brooks, M. N.; Carlson, T. K.; Bakthavachalu, B.; Schoenberg, D. R.; Torrelles, J. B.; Schlesinger, L. S., Mycobacterium tuberculosis lipomannan microRNA miR-125b. Proc Natl Acad Sci U S A 2011, 108 (42), 17408-13.

25. Wang, D.; Cao, L.; Xu, Z.; Fang, L.; Zhong, Y.; Chen, Q.; Luo, R.; Chen, H.; Li, K.; Xiao, S., MiR-125b reduces porcine reproductive and respiratory syndrome virus replication by negatively regulating the NF-kappaB pathway. PLoS One 2013, 8 (2), e55838.

26. Huang, H. C.; Yu, H. R.; Huang, L. T.; Chen, R. F.; Lin, I. C.; Ou, C. Y.; Hsu, T. Y.; Yang, K. D., miRNA-125b regulates TNF-alpha production in $\mathrm{CD} 14+$ neonatal monocytes via posttranscriptional regulation. J Leukoc Biol 2012, 92 (1), 171-82.

27. Chaudhuri, A. A.; So, A. Y.; Sinha, N.; Gibson, W. S.; Taganov, K. D.; O'Connell, R. M.; Baltimore, D., MicroRNA-125b potentiates macrophage activation. J Immunol 2011, 187 (10), 5062-8.

28. Kim, D. S.; Zhang, W.; Millman, S. E.; Hwang, B. J.; Kwon, S. J.; Clayberger, C.; Pagano, M.; Krensky, A. M., Fbw7gamma-mediated degradation of KLF13 prevents RANTES expression in resting human but not murine T lymphocytes. Blood 2012, 120 (8), 1658-67.

29. Kim, S. W.; Ramasamy, K.; Bouamar, H.; Lin, A. P.; Jiang, D.; Aguiar, R. C., MicroRNAs miR-125a and miR-125b constitutively activate the NF-kappaB pathway by targeting the tumor necrosis factor alpha-induced protein 3 (TNFAIP3, A20). Proc Natl Acad Sci U S A 2012, 109 (20), 7865-70.

30. Graff, J. W.; Dickson, A. M.; Clay, G.; McCaffrey, A. P.; Wilson, M. E., Identifying functional microRNAs in macrophages with polarized phenotypes. J Biol Chem 2012, 287 (26), 21816-25.

31. Li, D.; Kong, C.; Tsun, A.; Chen, C.; Song, H.; Shi, G.; Pan, W.; Dai, D.; Shen, N.; Li, B., MiR-125a-5p Decreases the Sensitivity of Treg cells Toward IL-6-Mediated Conversion by Inhibiting IL-6R and STAT3 Expression. Sci Rep 2015, 5, 14615.

32. Febbraio, M. A.; Pedersen, B. K., Muscle-derived interleukin-6: mechanisms for activation and possible biological roles. FASEB J 2002, 16 (11), 1335-47.

33. Sundararaj, K. P.; Samuvel, D. J.; Li, Y.; Sanders, J. J.; Lopes-Virella, M. F.; Huang, Y., Interleukin-6 released from fibroblasts is essential for up-regulation of matrix metalloproteinase1 expression by U937 macrophages in coculture: cross-talking between fibroblasts and U937 macrophages exposed to high glucose. J Biol Chem 2009, 284 (20), 13714-24.

34. Shoelson, S. E.; Lee, J.; Goldfine, A. B., Inflammation and insulin resistance. J Clin Invest 2006, 116 (7), 1793-801.

35. Mohamed-Ali, V.; Goodrick, S.; Rawesh, A.; Katz, D. R.; Miles, J. M.; Yudkin, J. S.; Klein, S.; Coppack, S. W., Subcutaneous adipose tissue releases interleukin-6, but not tumor necrosis factor-alpha, in vivo. J Clin Endocrinol Metab 1997, 82 (12), 4196-200.

36. Eder, K.; Baffy, N.; Falus, A.; Fulop, A. K., The major inflammatory mediator interleukin6 and obesity. Inflamm Res 2009, 58 (11), 727-36. 
37. Schultz, O.; Oberhauser, F.; Saech, J.; Rubbert-Roth, A.; Hahn, M.; Krone, W.; Laudes, M., Effects of inhibition of interleukin- 6 signalling on insulin sensitivity and lipoprotein (a) levels in human subjects with rheumatoid diseases. PLoS One 2010, 5 (12), e14328.

$498 \quad 38 . \quad$ Senn, J. J.; Klover, P. J.; Nowak, I. A.; Mooney, R. A., Interleukin-6 induces cellular insulin resistance in hepatocytes. Diabetes 2002, 51 (12), 3391-9.

39. Mauer, J.; Chaurasia, B.; Goldau, J.; Vogt, M. C.; Ruud, J.; Nguyen, K. D.; Theurich, S.; Hausen, A. C.; Schmitz, J.; Bronneke, H. S.; Estevez, E.; Allen, T. L.; Mesaros, A.; Partridge, L.; Febbraio, M. A.; Chawla, A.; Wunderlich, F. T.; Bruning, J. C., Signaling by IL-6 promotes alternative activation of macrophages to limit endotoxemia and obesity-associated resistance to 504 insulin. Nat Immunol 2014, 15 (5), 423-30.

505 40. Pedersen, B. K.; Steensberg, A.; Schjerling, P., Muscle-derived interleukin-6: possible 506 biological effects. J Physiol 2001, 536 (Pt 2), 329-37.

507 41. Sindhu, S.; Thomas, R.; Shihab, P.; Sriraman, D.; Behbehani, K.; Ahmad, R., Obesity Is a 508 Positive Modulator of IL-6R and IL-6 Expression in the Subcutaneous Adipose Tissue: Significance 509 for Metabolic Inflammation. PLoS One 2015, 10 (7), e0133494.

510 42. Esporcatte, B. L.; Tavares, I. M., Normal-tension glaucoma: an update. Arq Bras Oftalmol $5112016,79(4), 270-6$.

512 43. Fingert, J. H.; Robin, A. L.; Stone, J. L.; Roos, B. R.; Davis, L. K.; Scheetz, T. E.; Bennett, 513 S. R.; Wassink, T. H.; Kwon, Y. H.; Alward, W. L.; Mullins, R. F.; Sheffield, V. C.; Stone, E. M., Copy 514 number variations on chromosome $12 q 14$ in patients with normal tension glaucoma. Hum Mol 515 Genet 2011, 20 (12), 2482-94. 


\begin{tabular}{lcccc}
\hline \multicolumn{1}{c}{ Demographic characteristics } & GG (N=35) & GC (N=28) & CC (N=25) & P value \\
\hline Sex (n, \%) & & & & 0.421 \\
$\quad$ Male (54) & $23(42.6)$ & $16(29.6)$ & $15(27.8)$ & \\
$\quad$ Female (34) & $12(35.3)$ & $12(35.3)$ & $10(29.4)$ & \\
Age, years (mean, SD) & $65.3(5.3)$ & $64.8(8.2)$ & $65.5(7.5)$ & 0.625 \\
Systemic diseases (n, \%) & & & \\
$\quad$ Hypertension (55) & $28(50.9)$ & $16(29.1)$ & $11(20.0)$ & 0.356 \\
$\quad$ Diabetes mellitus (28) & $15(53.6)$ & $9(32.1)$ & $4(14.3)$ & 0.563 \\
$\quad$ Hyperlipidemia (26) & $15(57.7)$ & $8(30.8)$ & $3(11.5)$ & 0.835 \\
Body mass index, kg/m2 (mean, SD) & $25.6(5.5)$ & $24.9(6.2)$ & $24.5(7.8)$ & 0.642 \\
\hline Table 1. Demographic data of the subjects in this study & & &
\end{tabular}

Table 1. Demographic data of the subjects in this study 
A

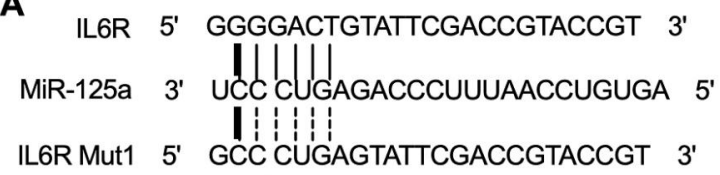

B

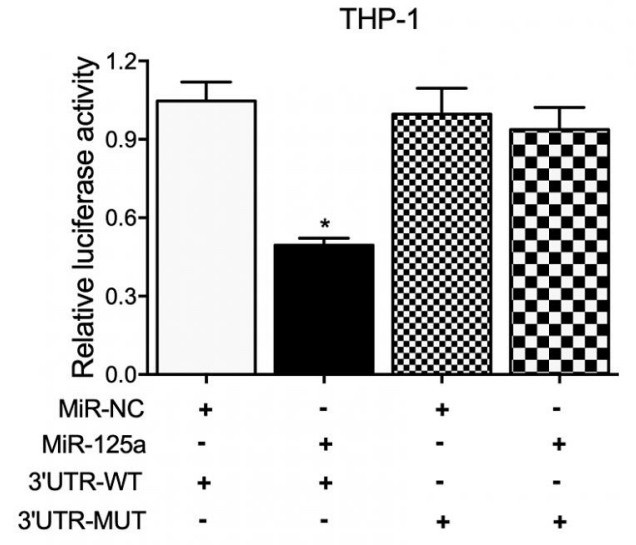

U937

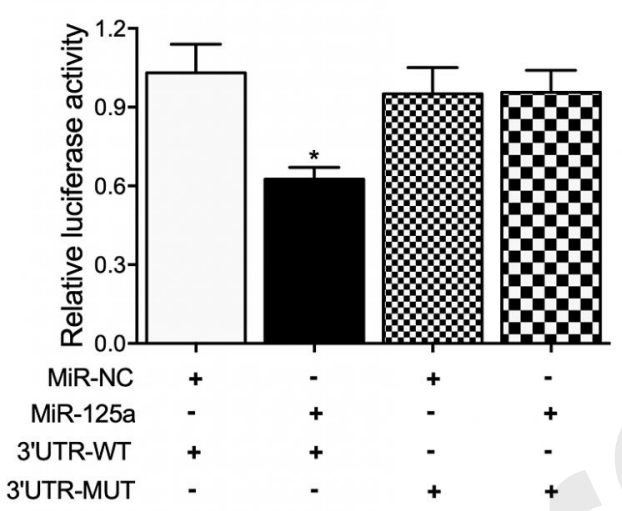

C

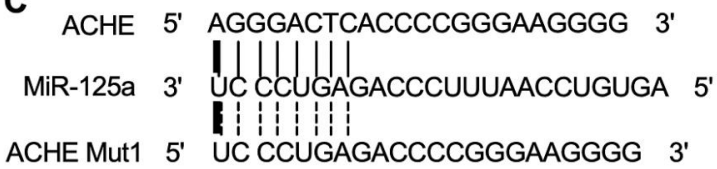

ACHE Mut1 5 ' UC CCUGAGACCCCGGGAAGgGg $3^{\prime}$
D
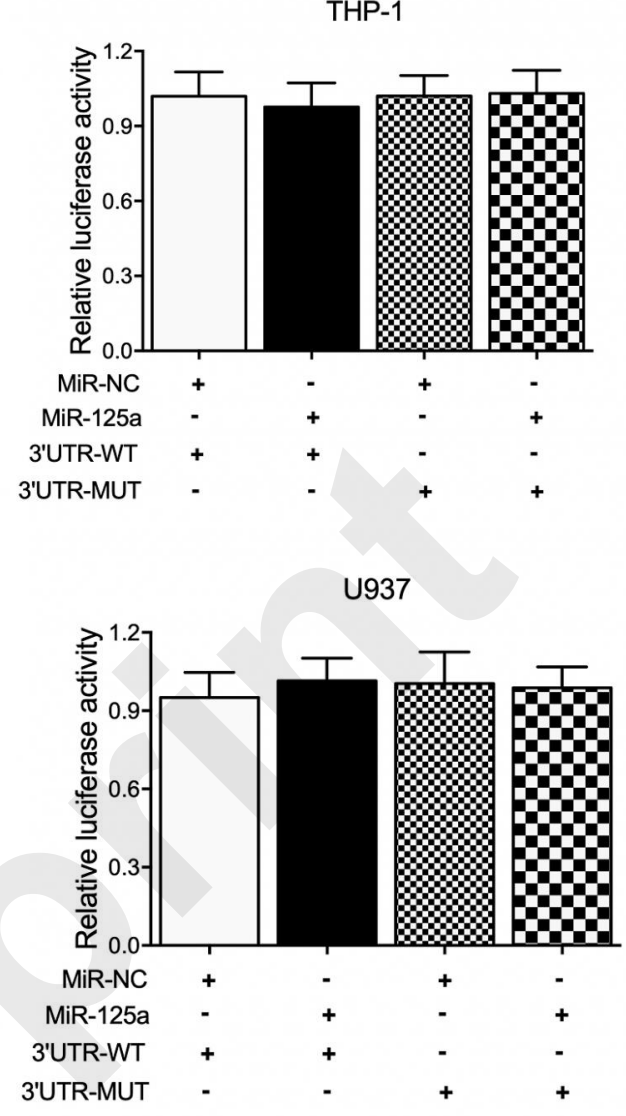

Fig.1

Luciferase assay confirmed the target genes of miR-125a in THP-1 and U937 cells ( ${ }^{*} \mathrm{P}$ value $<0.05$ vs. miR-NC group; number of replicas $=3$ )

A: predicted binding site of miR-125a in IL-6R

$\mathrm{B}$ : luciferase activities of miR-125a in THP-1 cells co-transfected with wild/mutant type of IL-6R and miR-125a /NC.

C: predicted binding site of miR-125a in ACHE

D: luciferase activities of miR-125a in THP-1 cells co-transfected with wild/mutant type of ACHE and miR-125a /NC. 

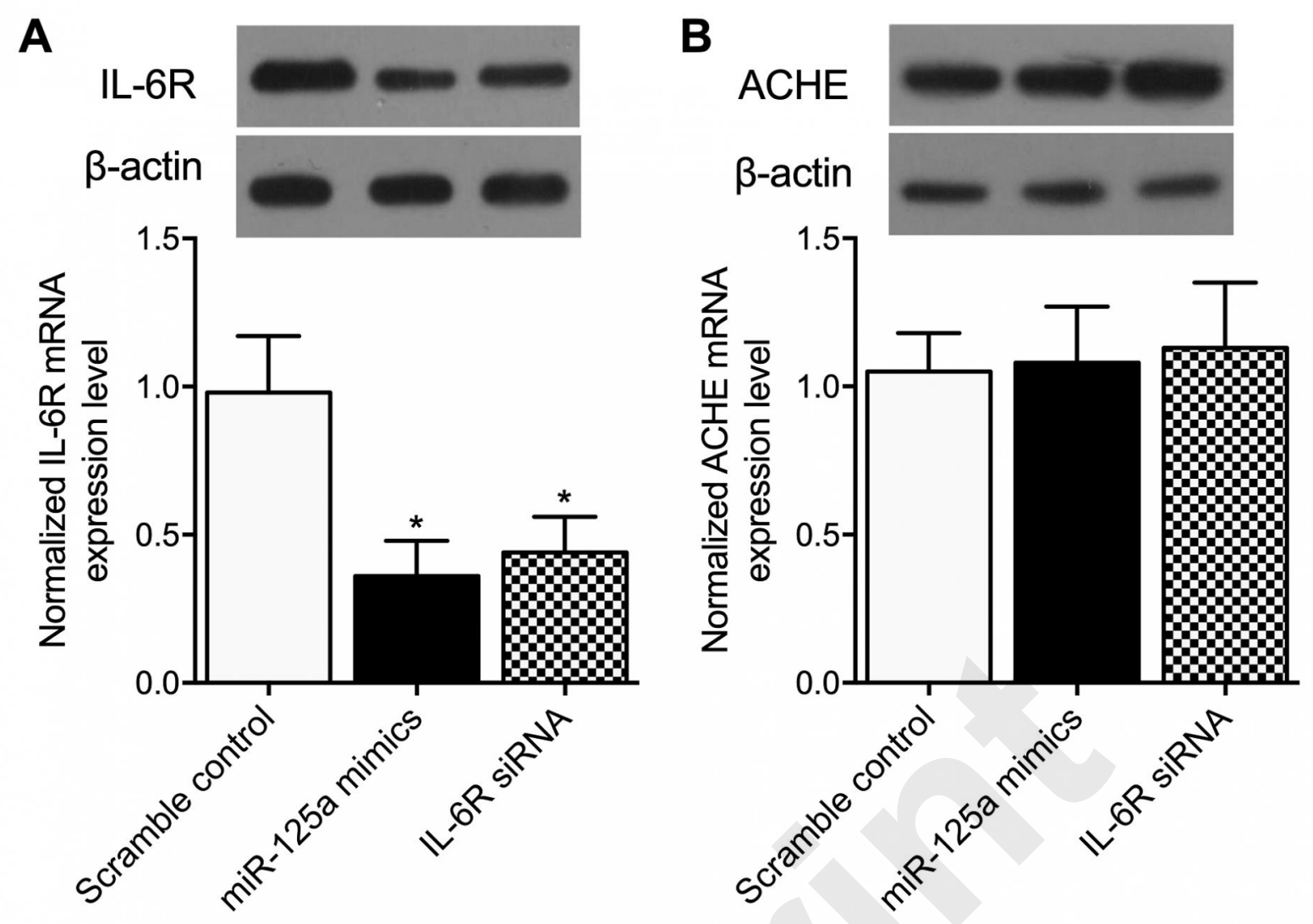

Fig. 2 mRNA and protein levels of IL-6R and ACHE in THP-1 cells transfected with NC, miR-125a mimics or IL-6R siRNA ( $P$ value $<0.05$ vs. scramble control group; number of replicas $=3$ ) A: mRNA and protein levels of IL-6R in the three THP-1 groups. B: mRNA and protein levels of ACHE in the three THP-1 groups. 
A
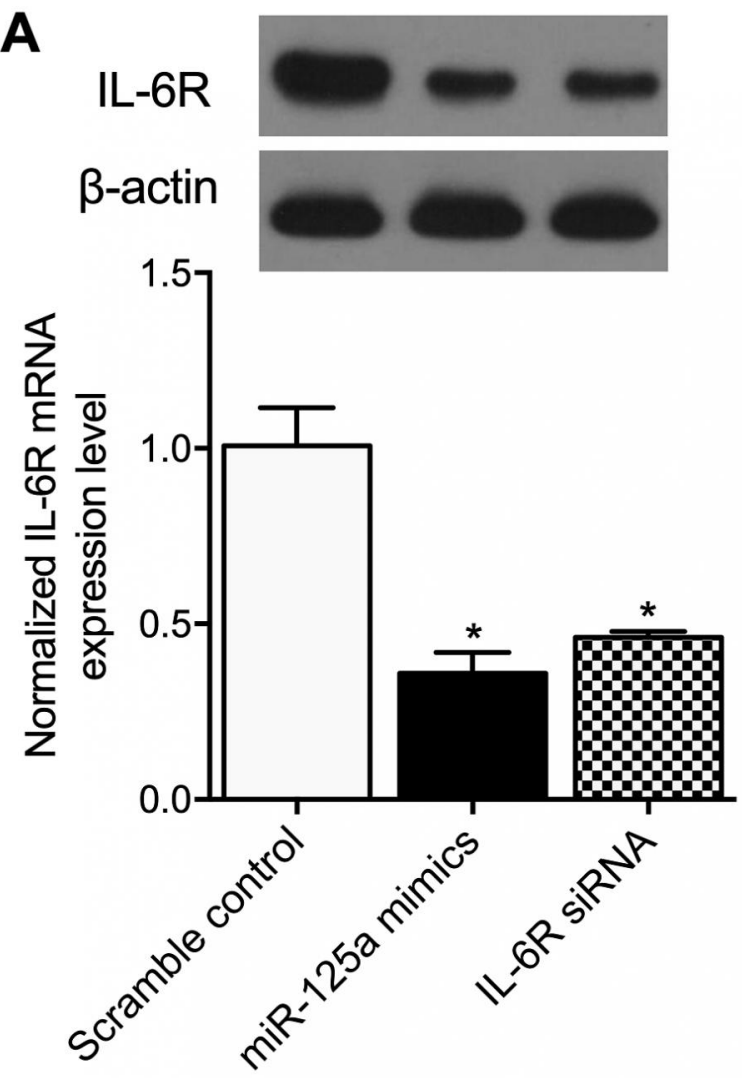

B
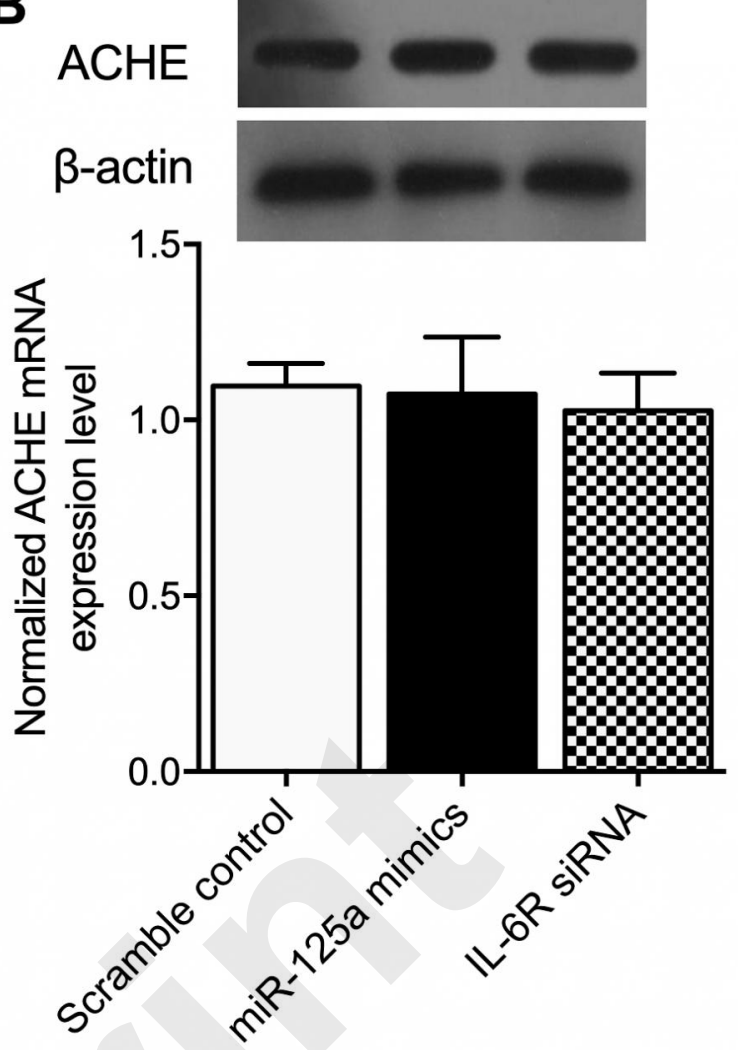

Fig. 3 mRNA and protein levels of IL-6R and ACHE in U937 cells transfected with NC, miR-125a mimics or IL-6R siRNA ( ${ }^{*} P$ value $<0.05$ vs. scramble control group; number of replicas $=3$ ) A: mRNA and protein levels of IL-6R in the three U937 groups. B: mRNA and protein levels of ACHE in the three U937 groups. 
A

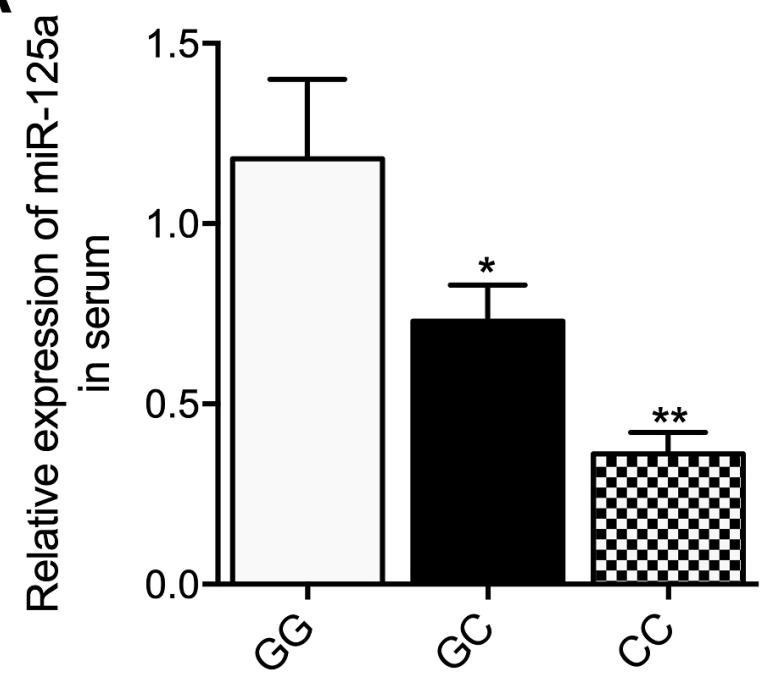

B

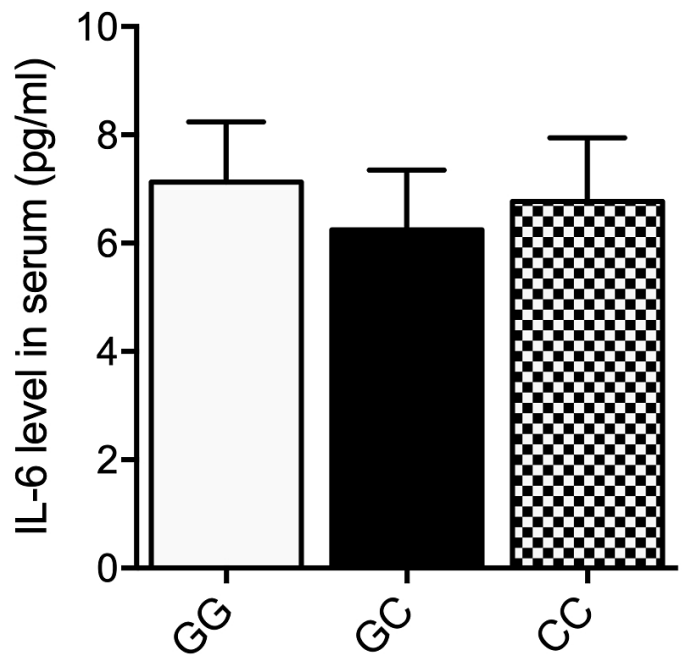

Fig.4

Relative expression of miR-125a mRNA and activity of IL-6R in blood samples collected from POAG patients carrying GG, GC, and CC genotypes of $r s 12976445$ ( ${ }^{*} P$ value $<0.05$ vs. GG group; ${ }^{* *} P$ value < 0.05 vs. GC group; number of replicas $=3$ ).

A: Relative expression of miR-125a in the three groups.

B: IL-6 activity in the three groups. 
A

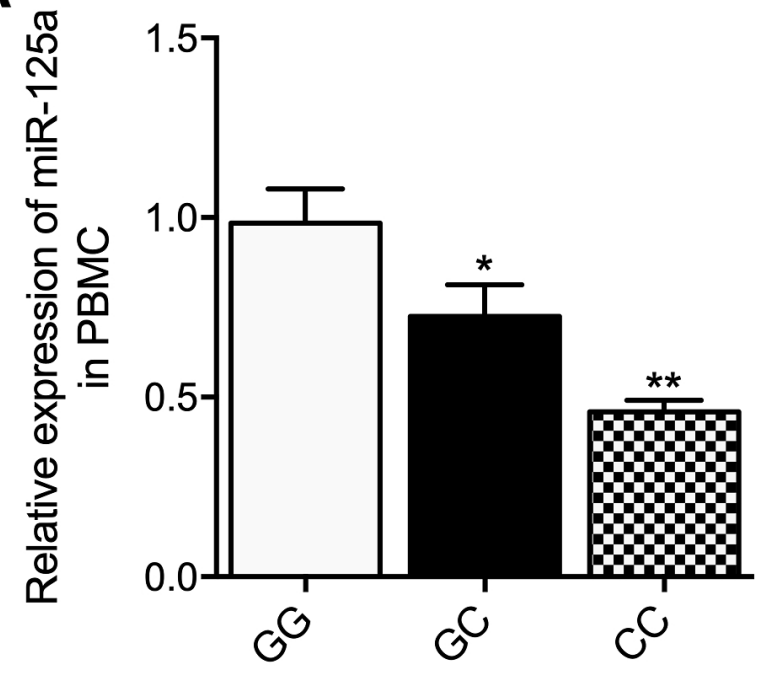

B

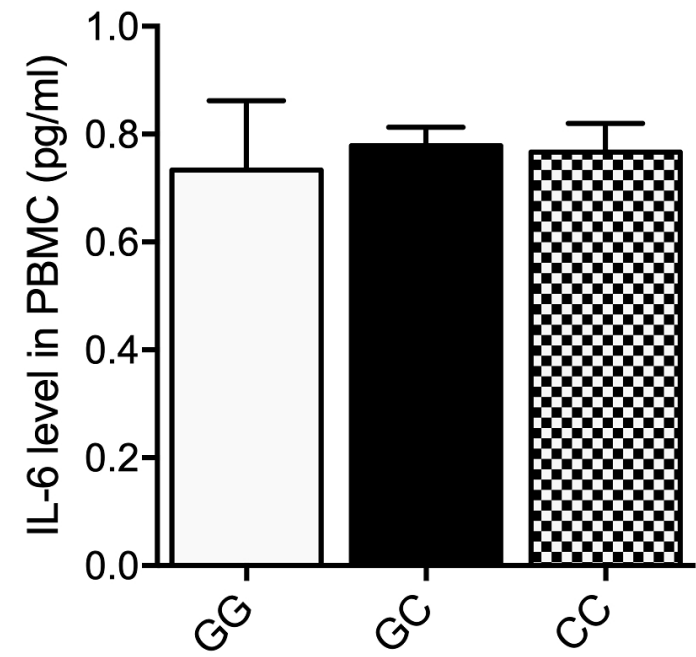

Fig.5

Relative expression of miR-125a mRNA and activity of IL-6R in PBMC samples collected from POAG patients carrying GG, GC, and CC genotypes of $r s 12976445$ ( ${ }^{*} P$ value $<0.05$ vs. GG group; ${ }^{* *} P$ value < 0.05 vs. GC group; number of replicas $=3$ ).

A: Relative expression of miR-125a in the three groups.

B: IL-6 activity in the three groups. 
A

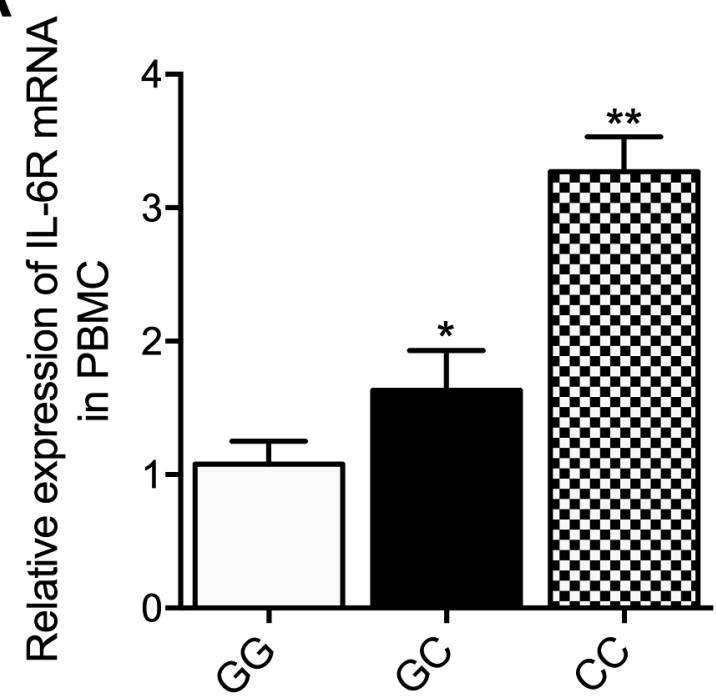

B

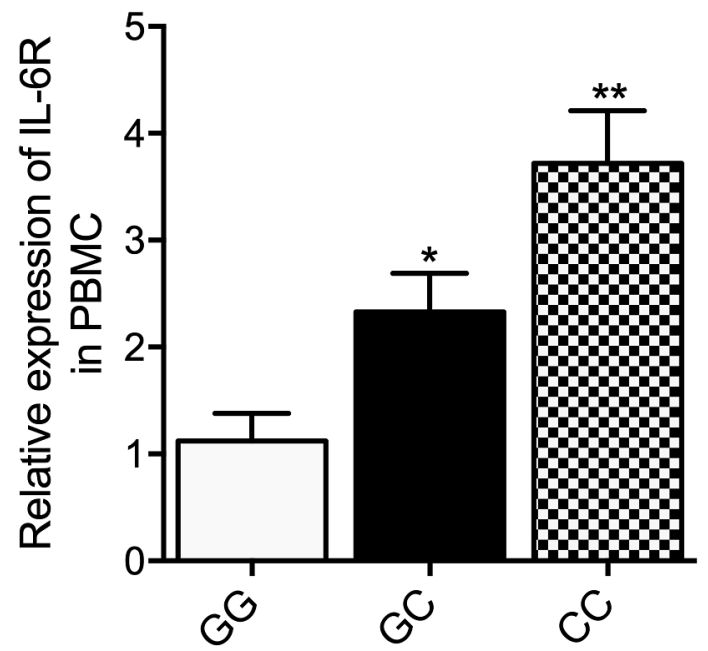

Fig.6

mRNA and protein levels of IL-6R in PBMC samples collected from POAG patients carrying GG, GC, and CC genotypes of rs 12976445 ( ${ }^{*}$ P value < 0.05 vs. GG group; ** $P$ value < 0.05 vs. $\mathrm{GC}$ group; number of replicas $=3$ ).

A: mRNA levels of IL-6R in the three groups

$B$ : protein levels of IL-6R in the three groups 
A

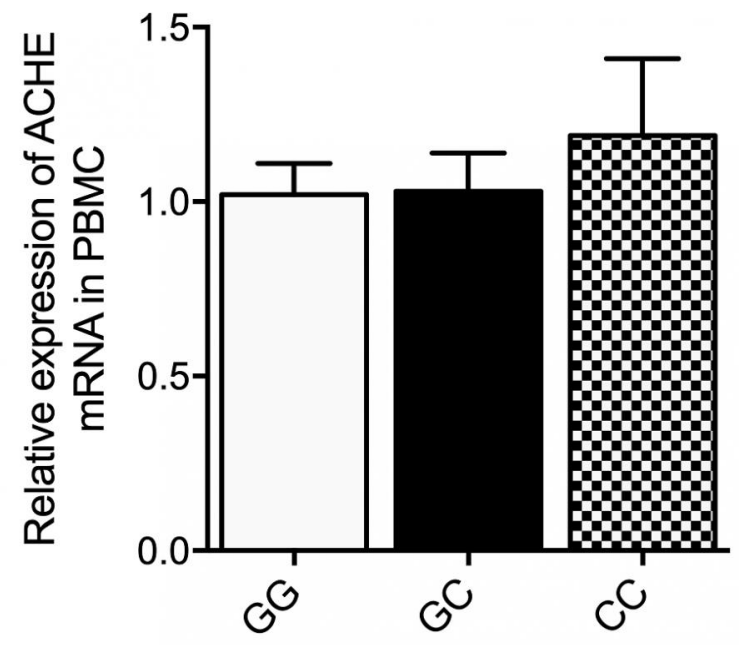

B

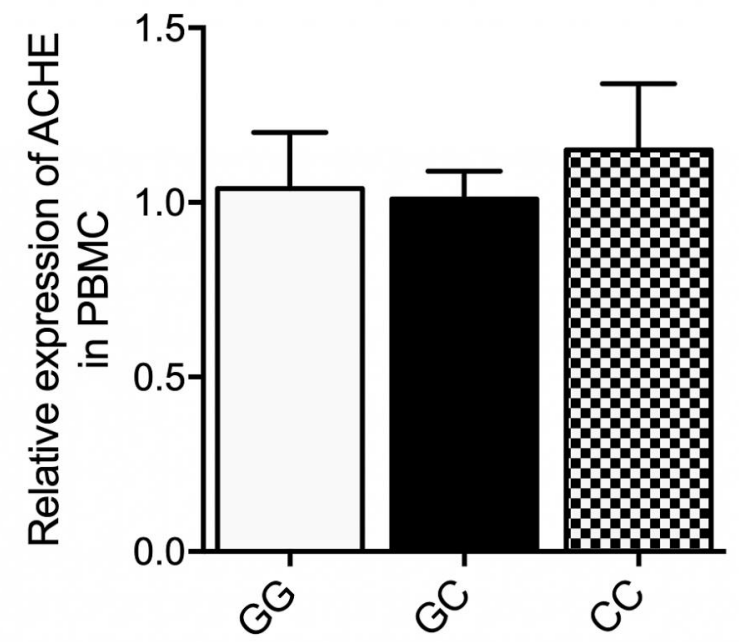

Fig.7

mRNA and protein levels of ACHE in PBMC samples collected from POAG patients carrying GG, GC, and CC genotypes of rs 12976445 (number of replicas = 3).

$A$ : mRNA levels of ACHE in the three groups

$B$ : protein levels of $A C H E$ in the three groups 


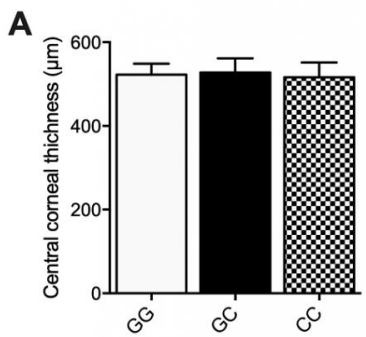

B

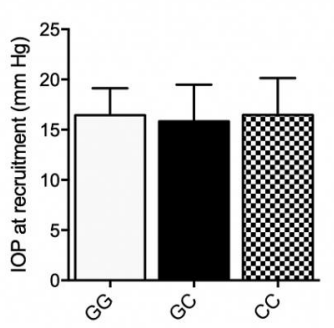

E

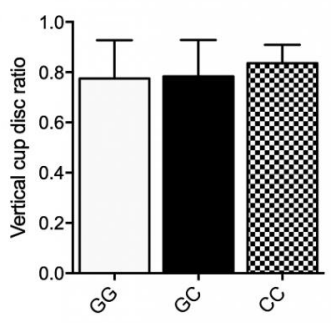

$\mathbf{F}$

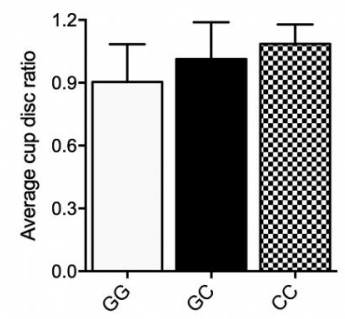

C

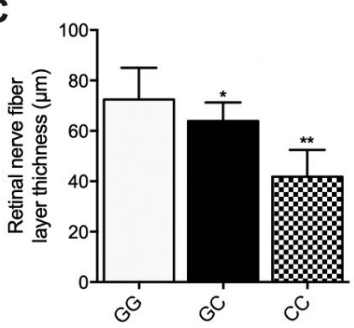

G
D

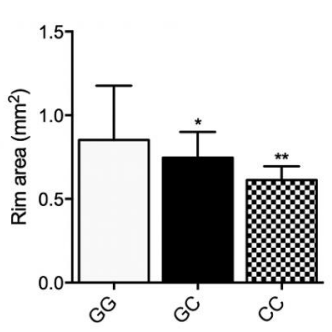

H

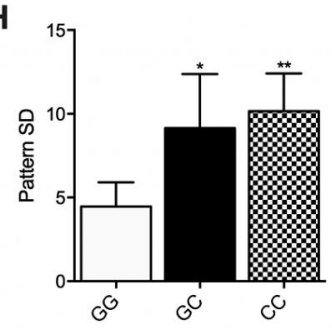

Fig.8

Comparison of demographic and clinical data among POAG patients carrying GG, GC and CC genotypes of rs 12976445 ( ${ }^{*} P$ value $<0.05$ vs. GG group; ${ }^{* *} P$ value $<0.05$ vs. GC group; number of replicas $=3$ ).

A: central corneal thickness in the three groups

$\mathrm{B}$ : IOP at recruitment in the three groups

C: retinal nerve fiber layer thickness in the three groups

D: rim area in the three groups

$\mathrm{E}$ : vertical cup disc ratio in the three groups

$\mathrm{F}$ : average cup disc ratio in the three groups

$\mathrm{G}$ : mean deviation in the three groups

$\mathrm{H}$ : pattern SD in the three groups 